11

\title{
Тороидальный резонатор с электростатической фокусировкой
}

\author{
(С) Т.Д. Дауменов, М.А. Хизирова
}

Алматинский университет энергетики и связи, 050000 Алматы, Казахстан

e-mail: dautl@mail.ru

(Поступило в Редакцию 9 апреля 2017 г. В окончательной редакции 5 сентября 2018 г.)

Рассмотрен вопрос о возможности использования одиночной линзы, встроенной в области сосредоточения электрического поля резонатора, для модуляции электронного потока по скорости. Показано, что применение одиночной линзы в качестве модулятора по скорости не влияет на величину коэффициента взаимодействия электронного потока с полем линзы.

DOI: 10.21883/JTF.2019.03.47182.2292

\section{Введение}

В электронике СВЧ для расчета и создания мощных усилителей и генераторов большое внимание уделяют формированию мощного электронного потока различной конфигурации и его транспортировке на значительные расстояния. Теории формирования электронных пучков большой интенсивности посвящен ряд исследований [1-3]. Известны способы транспортировки электронных потоков в приборах СВЧ с клистронным механизмом взаимодействия между входным и выходным резонаторами посредством различных статических электрических и магнитных полей [4]. Необходимость применения указанных полей обусловлена влиянием объемных зарядов и кулоновских сил расталкивания пространственного заряда на траекторию движения электронов. Как правило, фокусирующие действия электрических и магнитных полей осуществляются в дрейфовом пространстве СВЧ-приборов. В работе [5] показана возможность использования одиночной линзы для осуществления фокусировки потока электронов в вертикальном направлении в прямопролетном клистроне. Эта задача решена в первом приближении путем решения дифференциального уравнения для траектории электронов, движущихся в электростатическом поле с вращательной симметрией. Показано также, что длина дрейфового пространства $L$ должна равняться фокусному расстоянию одиночной линзы.

\section{Выражение для коэффициента взаимодействия потока электронов с СВч-полем одиночной линзы}

Рассмотрим трехэлектродную одиночную линзу, электроды которой состоят из трех плоских соосных диафрагм, расположенных на равном расстоянии $s$ друг от друга, параллельных между собой (рис. 1).
Осевое распределение электростатического потенциала $\Phi(z)$ представим в виде [6-8]

$$
\Phi(z)=\Phi_{0}\left(1-\frac{\chi^{2}}{1+\left(\frac{z-s}{d}\right)^{2}}\right),
$$

где $\Phi_{0}$ - потенциал свободного пространства, $\chi^{2}-$ параметр, изменяющийся в пределах $0<\chi^{2}<1, d-$ диаметр отверстий диафрагм. Начало координат совместим с плоскостью среднего электрода, а ось $z$ является осью симметрии системы. Пусть на крайние электроды одиночной линзы подается переменное напряжение с частотой $\omega$ и амплитудой $U_{m}$

$$
u=U_{m} \sin \omega t .
$$

Обозначим через $t_{0}$ момент прохождения некоторого электрона через центр среднего электрода линзы. Тогда, пренебрегая малым изменением скорости электрона внутри линзы, можно написать

$$
t=t_{0}+\frac{z}{\vartheta_{0}}
$$

Полная кинетическая энергия электрона, вошедшего в линзу с начальной скоростью

$$
\vartheta_{0}=\sqrt{\frac{2 e \Phi_{0}}{m}},
$$

на выходе из линзы имеет величину

$$
W=e \Phi_{0}+\Delta W
$$

где приращение кинетической энергии $\Delta W$ может быть представлено в следующем виде:

$$
\begin{aligned}
\Delta W= & \frac{e U_{m}}{2 s}\left[\int_{-s}^{s} \sin \left(\omega t_{0}+\frac{\omega z}{\vartheta_{0}}\right) d z\right. \\
& \left.+\frac{2 s}{\Phi_{0}} \int_{-s}^{s} \Phi^{\prime}(z) \sin \left(\omega t_{0}-\frac{\omega z}{\vartheta_{0}}\right) d z\right] .
\end{aligned}
$$




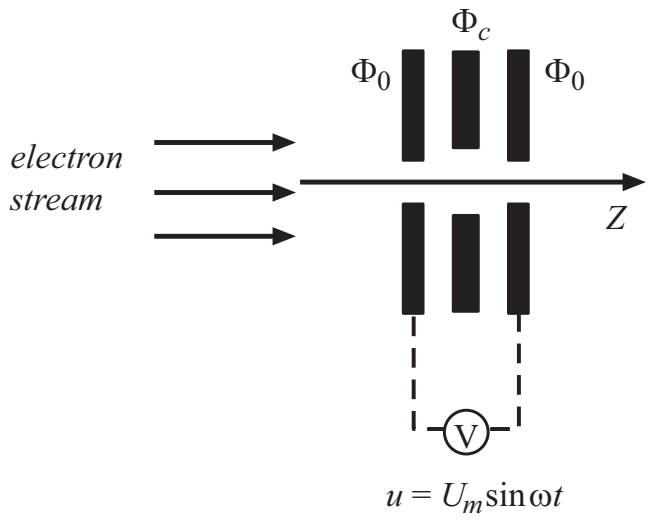

Рис. 1. Трехэлектродная одиночная линза. Переменное напряжение подается к крайним электродам.

Штрихи обозначают дифференцирование по координате $z$. После интегрирования приращение кинетической энергии может быть представлено в виде

$$
\begin{aligned}
\Delta W= & e U_{m}\left[M-\frac{\omega}{\vartheta_{0}} \chi^{2} I_{1}(z)\right] \sin \Omega t_{0} \\
& +\chi^{2}\left[-\sin \frac{\theta}{2}+\frac{\omega}{\vartheta_{0}} \chi^{2} I_{2}(z)\right] \cos \omega t_{0} .
\end{aligned}
$$

Здесь введены следующие обозначения:

$$
\begin{gathered}
I_{1}(z)=\int_{-s}^{s} f(z) \sin \frac{\omega z}{\vartheta_{0}} d z, \\
I_{2}(z)=\int_{-s}^{s} f(z) \cos \frac{\omega z}{\vartheta_{0}} d z, \\
f(z)=\left[1+\left(\frac{z}{d}\right)^{2}\right]^{-1} .
\end{gathered}
$$

Величина $\theta=\frac{2 \omega s}{\vartheta_{0}}$ характеризует невозмущенный угол пролета электронов через линзу, а через $M$ обозначен коэффициент взаимодействия электронного потока с переменным полем одиночной линзы

$$
M=\frac{\sin (\theta / 2)}{(\theta / 2)} .
$$

Проведенные расчеты показывают, что интеграл $I_{1}(z)$ обращается в нуль, т.е. применение одиночной линзы в качестве модулятора по скорости не влияет на величину коэффициента взаимодействия электронного потока с полем линзы. Во-вторых, ввиду малости половины невозмущенного угла пролета при выполнении условия

$$
s=\int_{-s}^{s} f(z) \cos \frac{\omega z}{\vartheta_{0}} d z
$$

сохраняется синусоидальный закон изменения приращения кинетической энергии

$$
\Delta W=e M U_{m} \sin \omega t_{0} .
$$

Тогда с учетом малости амплитуды переменного напряжения $\left(U_{m}<\Phi_{0}\right)$ по сравнению с потенциалами на крайних электродах одиночной линзы скорость электрона $\vartheta$ на выходе из линзы может быть представлена следующим образом:

$$
\vartheta \approx \vartheta_{0}\left(1+\frac{M U_{m}}{2 \Phi_{0}} \sin \omega t_{0}\right) .
$$

Таким образом, применение одиночной линзы в качестве модулятора электронного потока по скорости практически не меняет величину коэффициента взаимодействия электронного потока с полем линзы, однако наличие среднего электрода с потенциалом $\Phi_{c}$ дает возможность организовать объемную фокусировку электронного потока в вертикальном направлении.

\section{Недостатки входного резонатора клистрона классической схемы}

В объемном резонаторе роль сосредоточенной емкости играет плоский зазор в виде плоскопараллельных сеток в центре резонатора, роль сосредоточенной индуктивности - тороидальная поверхность. В СВЧприборах с клистронным механизмом взаимодействия в качестве входных и выходных устройств используются объемные резонаторы. Емкость входного резонатора находится под высоким положительным потенциалом, там же сосредоточено преимущественно электрическое высокочастотное поле. Под действием этого высокочастотного поля электронный поток модулируется по скорости, в дальнейшем переходя в модуляцию по плотности в дрейфовом пространстве СВЧ-прибора, а в выходном резонаторе снимают энергию модулированного электронного потока при вхождении в выходной резонатор в тормозящем режиме электрического поля.

Недостатками описанного выше входного резонатора является, во-первых, наличие сеток, являющихся механической преградой на пути потока электронов, вовторых, провисания эквипотенциальных поверхностей вблизи сеток, оказывающие рассеивающее действие на электроны. Эти факторы снижают величину конвекционного тока и тем самым понижают мощность СВЧприбора. Поэтому в традиционных схемах таких СВЧ приборов возникает необходимость использования различных транспортирующих систем в дрейфовом пространстве: системы однородного или неоднородного магнитного поля, системы однородного или неоднородного электрического поля. 


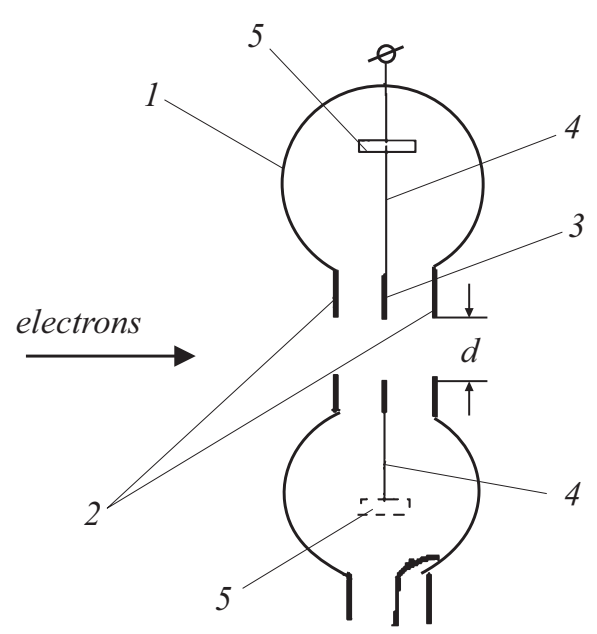

Рис. 2. Тороидальный резонатор с электростатической фокусировкой. Пояснения - в тексте.

\section{Преимущества предлагаемой схемы входного резонатора}

Положительный результат достигается тем, что вместо емкости, состоящей из двух параллельных сеток, где сосредоточено сверхвысокочастотное электрическое поле, используется одиночная линза, состоящая из трех диафрагм.

На рис. 2 представлена конструкция предлагаемого устройства [6]. Данное устройство представляет собой тороидальный резонатор 1 , в котором в области сосредоточения высокочастотного электрического поля установлена одиночная линза, состоящая из трех диафрагм с круглыми отверстиями равного диаметра, крайние электроды которой 2 находятся под одинаковым высоким ускоряющим потенциалом; на эти же электроды подается высокочастотное электрическое поле. Средняя диафрагма 3 устанавливается с помощью тонких проводящих нитей 4 , пропущенных через прорези 5 , параллельные линиям тока, расположенные на противоположных поверхностях тороида; на нее подается потенциал, отличающийся от потенциалов, поданных на крайние диафрагмы.

Совокупность трех диафрагм - это одиночная линза, фокусное расстояние которой регулируется потенциалом средней $\Phi_{c}$ диафрагмы.

Внешние электроды одиночной линзы находятся при потенциале $\Phi_{0}$, равном потенциалу окружающего линзу пространства, а потенциал $\Phi$ на внутреннем электроде может быть больше или меньше потенциала внешних электродов. Одиночная линза во всех случаях будет собирающей линзой [9]. Мы предполагаем, что $\Phi_{c}>\Phi_{0}$. Такой резонатор имеет возможность осуществлять вертикальную фокусировку электронного потока в приборах СВЧ с клистронным механизмом взаимодействия $[6,8]$.

\section{Заключение}

Таким образом, тороидальный резонатор предлагаемой конструкции может выполнять одновременно роль модулятора электронов по скорости, с одной стороны, и транспортирующей системы в дрейфовом пространстве, с другой, и поток сгустков электронов может быть сфокусирован на поверхности входного окна выходного резонатора. Такой резонатор с электростатической фокусировкой способствует повышению мощности СВЧприборов клистронного типа за счет увеличения конвекционного тока.

\section{Список литературы}

[1] Sushkov A.D., Molokovsky S.L. Investigation of electron guns with electron beam current control. 3-Czechosl. Conf. on electronics and vacuum. Phys. Transactions. Prague., 1967. P. 577-588.

[2] Овчаров В.T. // РЭ. 1968. № 8. С. 1367-1378.

[3] Кормилицин Б.Т., Овчаров В.Т. // РЭ. 1960. № 7. С. 1112 1117.

[4] Молоковский С.И., Сушков А.Д. Интенсивные электронные и ионные пучки. М. 1991. 304 с.

[5] Дауменов Т., Хизирова М.А. // Изв. вузов. Физика. 2016. T. 5. № 1. C. 16-19.

[6] Патент РК № 23239. Дауменов T. Тороидальный резонатор с электростатической фокусировкой.

[7] Дауменов T. // Uzbek J. Physics. 2009. T. 11. № 1. C. 47-51.

[8] Дауменов T. // Тезисы доклада к девятому всероссийскому семинару „Проблемы теоретической и прикладной электронной и ионной оптики““. М. 2009. 62 с.

[9] Кельман В.М., Явор С.Я. Электронная оптика. Л.: Наука, 1968. $488 \mathrm{c}$. 